\title{
Erstversorgung im Katastrophenfall: Beiträge der Armee und des Koordinierten Sanitätsdienstes (KSD)
}

\section{Gianpiero A. Lupi*}

* Dr. med., Divisionär, Chef Sanität/ Oberfeldarzt der Armee und Beauftragter des Bundesrates für den KSD

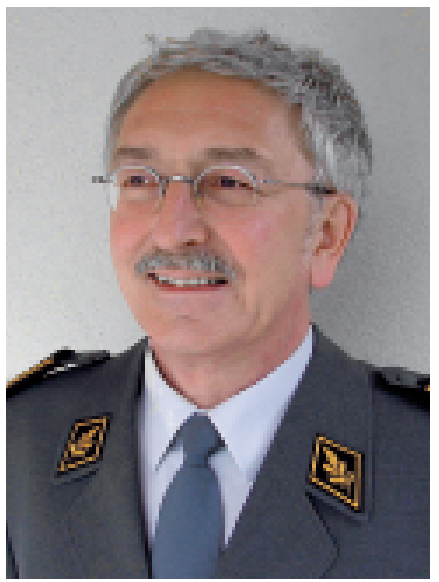

Gianpiero A. Lupi
Bei einem Patientenanfall, der schon in der prähospitalen Stufe mit den ordentlichen Abläufen im Gesundheitswesen nicht mehr bewältigt werden kann, werden in der Regel koordinative Führungsmassnahmen auf regionaler oder kantonaler Ebene getroffen. In dieser Phase kann die Armee sogenannte «Spontanhilfe» leisten. Dies bedeutet, dass Sanitätsformationen, die im Rahmen einer regulären Dienstleistung (Rekrutenoder Kaderschulen, Wiederholungskurse) bezüglich ihres Leistungsprofils und ihres Standortes geeignet sind, zeitgerecht die zivilen Einsatzkräfte zu unterstützen, dies anbieten oder auf Anfrage ohne weitere Formalitäten in der Verantwortung des vor Ort zuständigen Kommandanten tun können und sollen.

Ist das zivile Gesundheitswesen trotz der genannten Massnahmen und interkantonaler Zusammenarbeit in der Ereignisbewältigung überfordert, dann können die Kantone die subsidiäre Unterstützung durch die Armee beantragen, die dafür auch über Sanitäts- und Spitalformationen in verschiedenen Bereitschaftsgraden verfügen. Aus Platzgründen wird hier nicht auf Einzelheiten eingegangen.

Ein neuer und innovativer Beitrag der Armee und des Koordinierten Sanitätsdienstes (KSD), der in Ärztekreisen noch wenig bekannt ist, soll aber hier etwas ausführlicher vorgestellt werden.

Gemäss Art. 4d der Verordnung über den KSD kann der Beauftragte des Bundesrates für den KSD eine aktualisierte Gesamtübersicht über verfügbare Ressourcen im Gesundheitswesen Schweiz erstellen und diese den KSD-Partnern für die Vorbereitung und den Einsatz zur Verfügung stellen. Dies geschah bis vor einigen Jahren mit der periodischen Publikation der sogenannten «Liste der Spitäler», die aber von begrenzter Aktualität und Entscheidungshilfe im Alltag und in besonderen und ausserordentlichen Lagen war.

Mit dem IES-KSD (Informations- und EinsatzSystem KSD) entsteht jetzt ein internetbasiertes System, das den autorisierten KSD-Partnern einen Online-Zugriff auf alle aktuellen und zur Ereignisbewältigung entscheidenden Ressourcendaten des Systems Gesundheit und Sanität bietet und einen Informations- und Führungsdialog zwischen allen beteiligten Instanzen erlaubt.

Bei Unfällen, in Krisensituationen, Notlagen und Naturkatastrophen ist ein reibungslos funktionierender und koordinierter Sanitätsdienst im wahrsten Sinn des Wortes «überlebenswichtig». Er hilft den Rettungskräften, die Übersicht zu behalten und die richtigen Entscheidungen zu treffen. Wie viele Verletzte gibt es? Welche Einsatzkräfte stehen wie schnell zur Verfügung? Welche Spitäler können Patienten kurzfristig aufnehmen und wo können welche Verletzungen optimal versorgt werden? Schnelle Information und Kommunikation sind die wichtigsten Erfolgsfaktoren. Die zentrale Aufgabe des KSD besteht darin, den Einsatz aller zivilen und militärischen Stellen, die mit sanitätsdienstlichen Massnahmen beauftragt sind, zu unterstützen und stufengerecht zu koordinieren. Ziel ist eine optimale Nutzung und Auslastung der personellen, materiellen und infrastrukturellen Kapazitäten, damit alle Patienten in jeder Situation bestmöglich sanitätsdienstlich versorgt werden können.

Auf das zentrale Informations- und EinsatzSystem (IES-KSD) haben Einsatzzentralen und Spitäler aller Kantone Zugriff. Standardisierte Einsatzprozesse und ein automatisierter Workflow sollen dafür sorgen, dass die Abläufe weiter beschleunigt werden und die Rettungskräfte noch schneller und effizienter arbeiten können. Darüber hinaus hat der KSD eine Online-Datenbasis geschaffen, die alle Kantone mit Informationen über Best-Practice-Prozesse für potentiell gefährliche Situationen versorgt. Und schliesslich ermöglicht das System auch ein landesweites Tracking von Patienten, damit Angehörige jederzeit über Aufenthaltsort und Befinden verletzter oder vermisster Familienmitglieder informiert werden können.

Das Informations- und Einsatz-System (IESKSD) unterstützt den Austausch von Echtzeitinformationen zwischen allen im Rahmen des KSD miteinander kooperierenden Stellen. Über eine rollenbasierte komfortable Portaloberfläche lassen sich beispielsweise aktuell verfügbare Betten in der Notfallaufnahme, medizinische Geräte 


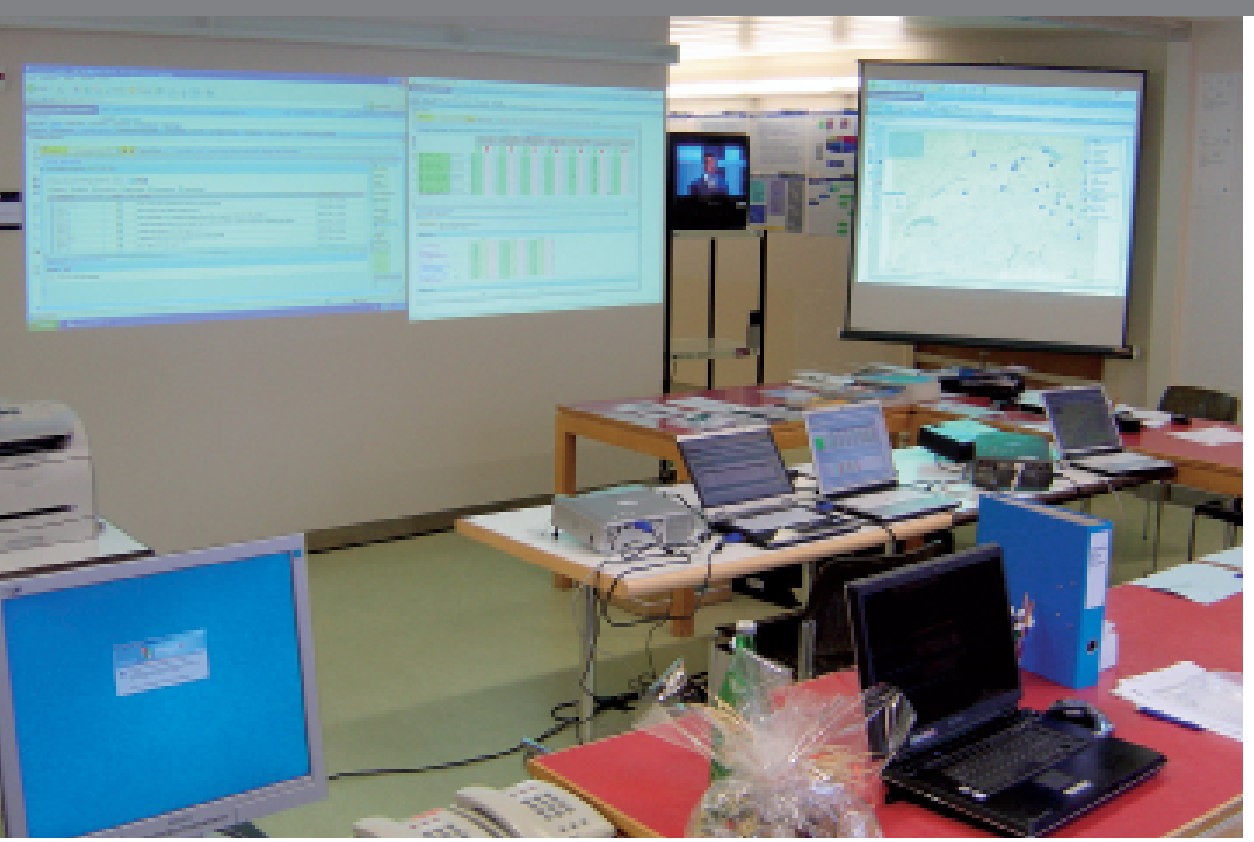

Das zentrale Informations- und Einsatz-System (IES-KSD) im Echteinsatz am World Economic Forum in Davos.

\section{Nutzen für Einsatzzentralen:}

- Ortsunabhängiger gesicherter Internetzugang auf die IES-Plattform

- Abbilden eigener Dispositive für den schnellen Zugang auf sanitätsdienstliche Ressourcen

- Einfache parallel auslösbare Kommunikation (FAX, E-Mail, SMS, Pager und Voice-Meldungen) zu Spitälern und anderen Institutionen

- Effiziente Ressourcensuche mit Resultatsdarstellung in Listen und grafischen Karten

- Übersicht über verfügbare sanitätsdienstliche Ressourcen im eigenen Zuständigkeitsbereich

- Gesamtschweizerische Übersicht über freie Kapazitäten im Gesundheitswesen

\section{Nutzen für Spitäler:}

- Dezentraler Web-Zugang für alle autorisierten Personen

- Verbesserter Informationsfluss mit den Einsatzzentralen

- Erhalt von Voralarmen, Alarmen, Lageberichten und Entwarnung

- Frühzeitige Information über die Patientenzuweisung

- Frühzeitige Information als Entscheidungsgrundlage für das Auslösen des Katastrophenplans

- Einfache strukturierte Rückmeldung der Notfallaufnahmekapazitäten
IES die Einsatzzentralen, Rettungsdienste und Spitäler stets zuverlässig über die aktuellste Lage.

Für das Rettungs- und Sanitätswesen bedeutet IES einen wichtigen Schritt nach vorne. Denn vor der Implementierung von IES waren sanitätsdienstliche Informationen ausschliesslich dezentral verfügbar, jeder Kanton koordinierte seine Einsätze autonom. In Notfallsituationen verstrich daher oftmals einige Zeit, ehe kantonsübergreifend alle erforderlichen Ressourcen mobilisiert waren. Unter anderem, weil die Einsatzzentrale zunächst telefonisch in jedem Spital der Nachbarkantone verfügbare Betten und Ressourcen abfragen musste. Erschwerend kam hinzu, dass der Kostendruck im Gesundheitswesen auch in der Schweiz zu einem Abbau von Kapazitäten und Reserven geführt hat und deshalb spezielle Behandlungsmöglichkeiten nur noch in ausgewählten Spitälern vorhanden sind.

Gehen Notrufe ein, gibt ein geschulter Operator diese mit allen verfügbaren Details direkt über die Portaloberfläche in das System ein. Festgehalten werden beispielsweise die Art des Notfalls, die Zahl der Verletzten sowie Details zu den einzelnen Verletzungen. Anschliessend kann die Einsatzzentrale direkt über IES Rettungsfahrzeuge und Einsatzkräfte disponieren - entsprechende Mitteilungen können als Fax, E-Mail, SMS oder Pagernachrichten versendet werden. Auch die Kommunikation zwischen den verschiedenen Einsatzzentralen lässt sich direkt über IES abwickeln. Leistungsfähige Suchfunktionen erleichtern dem Notfallpersonal zudem die Entscheidung, wohin die Verletzten transportiert werden sollen. Integrierte geographische Informationen zu Standorten und Wegen sorgen dabei für eine rasche Orientierung. Über das IES kann innert kürzester Zeit das Spital gefunden werden, das die medizinischen Bedürfnisse eines Patienten am besten erfüllt und am schnellsten erreichbar ist.

IES schafft auch für die angeschlossenen Spitäler einen wertvollen Zeit- und Informationsvorsprung: Sie werden bei einem ausserordentlichen Ereignis frühzeitig alarmiert und mit elektronischen Lageberichten laufend über die aktuelle Lageentwicklung informiert. Wenn keine weiteren Patienten mehr zu erwarten sind, kann ebenso rasch wieder die Entwarnung erfolgen. Umgekehrt können die Spitäler über das Internet oder über einen entsprechenden Webservice ihre aktuellen Notfallaufnahmekapazitäten an die Rettungszentralen melden. Der Abgleich von Bedarf und vorhandenen Kapazitäten kann automatisiert erfolgen. So lässt sich zusätzlicher manueller Datenerfassungsaufwand vermeiden. Spital transportiert werden. Zudem informiert sowie Spezialisten in den Spitälern mit dem Bedarf von Sanitätsnotrufzentralen und Rettungsdiensten online abgleichen. Vorbei sind Zeiten, als Notrufverantwortliche zun in den Spitälern teilweise erst aus den Radiooder Fernsehnachrichten erfuhren, was gerade auf sie zukommt. Patienten und Unfallopfer können dadurch schneller erstversorgt und anschliessend sofort in ein optimal geeignetes 
Zusätzlich bietet IES die Möglichkeit, bewältigte Notfall- und Krisensituationen zu analysieren und aufzuarbeiten: Die Führungsstäbe und Aufsichtsbehörden können die Reporting- und Analysefunktionen nutzen, um wichtige Kennzahlen zu vergleichen. Dabei können Daten aus SAP-Lösungen und -Anwendungen ebenso betrachtet werden wie Werte aus anderen Datenquellen - beispielsweise, um die Erfüllung von Vorgaben zu messen oder anfallende Kosten mit verfügbaren Budgets abzugleichen. Auf diese Weise lassen sich Rettungsszenarien und -prozesse hinsichtlich ihrer Leistungsfähigkeit und Wirtschaftlichkeit steuern und verbessern.

Zum Echteinsatz von IES im Rahmen der World-Economic-Foren 2005, 2006 und 2007 waren jeweils bereits über zehn Spitäler angeschlossen. In naher Zukunft kann das System überall in der Schweiz von autorisierten Verantwortlichen aller KSD-Partner genutzt werden. Die Geschäftsstelle KSD hat im Rahmen der Pandemievorbereitung des Bundes bereits damit begonnen, schrittweise alle Akutspitäler (ca. 220) anzubinden und IES so als Standardsystem für das tägliche medizinische Notfallmanagement zu etablieren. Als umfassende Informations- und Kommunikationszentrale für alle an der Notfallversorgung beteiligten Partnerorganisationen hilft IES dann, den gesamten Verwaltungsaufwand $\mathrm{zu}$ reduzieren und die Kosten zu senken.
Ländergrenzen lassen sich mit dem webbasierten System mühelos überwinden: Auch die Organisatoren der gemeinsam von der Schweiz und Österreich auszurichtenden Fussball-EM 2008 sind bereits an den KSD herangetreten! Ziel ist, dass IES bis zur EM schweizweit eingeführt ist. IES hat das Potential, in unterschiedlichsten Bereichen Verwendung zu finden. Auch ausserhalb des Sanitätsdienstes sind bereits Einsatzkräfte, Verantwortliche, Behörden und Institutionen zunehmend auf den hohen Nutzen und die vielfältig einsetzbaren, umfassenden Funktionalitäten von IES aufmerksam geworden! Beweis dafür liefern die Schweizerischen Bundesbahnen SBB und die Polizeikorps der Kantone Bern, Aargau und Solothurn, bei welchen das Modul «Patientenleitsystem/Personen- und Patientenerfassung» (PLS-PPE) bereits im Einsatz ist.

Die «Luzerner Trendtage Gesundheit» thematisieren Perspektiven und Trends im Gesundheitswesen. Sie stellen eine Plattform des Wissenstransfers und der Meinungsbildung bereit und wollen damit Entscheidungsgrundlagen für ein effizientes sowie gesellschaftlich und finanziell tragbares Gesundheitswesen in der Schweiz bereitstellen. Darüber hinaus sollen sie eine jährliche Standortbestimmung zu ausgewählten Fragen über das nationale und gegebenenfalls auch das internationale Gesundheitswesen ermöglichen. Der Kongress richtet sich an Entscheidungsträger aller wichtigen Bereiche des Gesundheitssystems.

\section{U ZERNER $\quad$ 3. Luzerner Trendtage - TRENDTAGE - G E S U N D H E I T \\ 3. Luzerner \\ Mi/Do, 21./22. März 2007, KKL Luzern}

\section{Altius - citius - fortius: \\ 3. Luzerner Trendtage Gesundheit 2007}

Der Treffpunkt, wenn es um die Zukunft im Gesundheitswesen geht

Datum: Mittwoch, 21., und Donnerstag, 22. März 2007

Ort: KKL Luzern, Luzerner Saal

Thema: «Notfallmedizin». Im Mittelpunkt stehen drei Themenschwerpunkte:

- Erstversorgung und Notfallmedizin allgemein

- Herz und Kreislauf

- Schmerz und Doping

Anmeldung: Unter www.trendtage-gesundheit.ch

Rubrik Trendtage/Anmeldung

Anmeldeschluss ist der 19. März 2007 